\title{
Risk Perception for Developing Erectile Dysfunction Among Malaysian Men with Type 2 Diabetes Mellitus
}

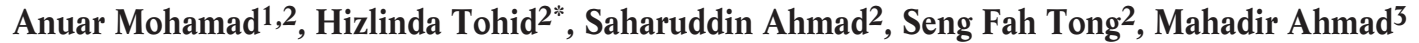

\author{
${ }^{1}$ Sungai Besi Health Clinic, Ministry of Health Malaysia, Kuala Lumpur, Malaysia \\ 2Department of Family Medicine, Faculty of Medicine, Universiti Kebangsaan Malaysia, Kuala Lumpur, Malaysia \\ ${ }^{3}$ Clinical Psychology and Behavioral Health Programme, Faculty of Health Sciences, Universiti Kebangsaan Malaysia, Kuala Lumpur, Malaysia
}

\begin{abstract}
Risk perception for developing erectile dysfunction (ED) is an appreciation of the susceptibility to having ED and its severity. This study examined this risk perception and its associated factors among 180 men with type 2 diabetes mellitus (T2DM), who claimed not to have ED. This cross-sectional study was conducted at a public health clinic using a validated self-administered questionnaire, which assessed participant characteristics, perceived susceptibility to developing ED, perceived severity of ED, and knowledge on risk factors for ED. About $71.1 \%$ had an inaccurate perception of susceptibility to developing ED and their perception on its severity was moderate (median (IQR) score: 10.0 (6.0); range score: 3-15; midpoint: 9). In multiple linear regression, having ED symptoms ( $p$-value $<0.001$ ) and secondary ( $p$-value $=0.045$ ) or tertiary education ( $p$-value: 0.022$)$ significantly contributed to a higher perception of susceptibility. A higher perception of severity was significantly found in Malays ( $p$-value $<0.001$ ), the employed ( $p$-value $=0.026$ ), and those with better knowledge on risk factors for ED ( $p$-value < 0.001). Risk perception for developing ED among men with T2DM appears poor and it was significantly influenced by sociocultural factors, educational attainment, ED symptoms, and knowledge on risk factors for ED. Thus, to improve their risk perception, they should be provided appropriate counseling and education.
\end{abstract}

Keywords: Diabetes mellitus, disease susceptibility, erectile dysfunction, perception, risk

\section{Introduction}

Erectile dysfunction (ED) is defined as the inability to achieve and/or maintain a penile erection sufficient for satisfactory sexual intercourse. ${ }^{1}$ The global prevalence of ED for all age groups ranges between $13 \%$ and $21 \% .^{2}$ As ED is commonly considered a loss of manhood, patients usually suffer significant psycho-social impacts such as depression, anger, and guilt of letting down their partner, which can contribute to a low quality of life (QOL). ${ }^{3}$ However, some men attribute ED to the normal process of aging and fate. ${ }^{4}$ Erectile dysfunction is more prevalent among patients with type 2 diabetes mellitus (T2DM). In Malaysia that prevalence is up to $89.2 \% .^{5}$ These patients are more likely to have severe ED, which is less responsive to medical treatment and leads to lower QOL.6,7

Due to its significance, risk perception for developing ED is important to evaluate because it potentially affects their help-seeking behavior. Risk perception is one's belief about a risk (potential harm or loss) through subjective judgment, and it is a construct of the mind. ${ }^{8}$ It is a core component of the Health Belief Model (HBM), influencing behavioral change. It comprises two domains: (1) perceived susceptibility and (2) perceived severity. According to the HBM, individuals who feel susceptible to health threats would regard the potential outcomes as severe and would take action to reduce the risk. ${ }^{8}$ In this context, theoretically, when a patient with T2DM feels threatened to have diabetic complications, including ED, he will take precautions and implement actions to reduce his risk by taking steps to achieve optimal glycaemic control to slow down or halt complications of diabetes. A randomized controlled trial conducted among 526 men with T2DM found that participants who perceived themselves at risk of having diabetes-related complications showed better self-care behavior. ${ }^{9}$ Besides, participants with high levels of risk knowledge and low levels of optimistic bias showed significant dietary, exercise, and medication adherence changes. ${ }^{9}$ In the previous study, optimistic bias was defined as one's belief of having less chance to develop diabetes-related complications than those with similar characteristics. ${ }^{9}$ Similarly, Pereira, et
Correspondence *: Hizlinda Tohid, Department of Family Medicine, Faculty of Medicine, Universiti Kebangsaan Malaysia, Bandar Tun Razak, Cheras, 56000 Kuala Lumpur, Malaysia, E-mail: hizlinda2202@gmail.com, Phone: +601-9222 2109
Received : September 03, 2020

Accepted : January 03, 2021

Published: February 20, 2021 
al. ${ }^{10}$ showed that those with a higher perception of having diabetes-related complications tended to have better foot care adherence.

However, the risk perception for diabetes-related complications was generally low, as shown in a recent systematic review of 18 studies. ${ }^{11}$ In this review, the studies were performed among men with T2DM in developed countries, and the risk perception assessed was mainly for cardiovascular and eye complications. The review also highlighted the presence of optimistic bias that could lead to their poor risk perceptions. ${ }^{11}$ However, how men with T2DM perceive their risk of having ED has not been well studied in Malaysia. A local study related to ED risk perception noted that men with T2DM perceived a higher impact of ED on QOL than non-diabetics. ${ }^{12}$ This study indicated that men with diabetes might perceive the seriousness of ED.

The present study aimed to determine the risk perception of ED among men with T2DM through examining their perceived susceptibility to develop ED and their perceived severity of ED. This study also aimed at determining factors associated with their risk perception. Information obtained from the research could provide a baseline understanding of how Malaysian men with T2DM perceive their risk of acquiring ED. This information would help to design concerted health education to improve their risk perception, resulting in better illness perception and adherence to self-care management.

\section{Method}

This study was a cross-sectional study conducted at a public health clinic in Selangor, Malaysia, between August and October 2019. This clinic provides primary care service to 150,000 people of the surrounding communities. About 5,638 patients with T2DM are followed up by a dedicated team trained to provide personalized and comprehensive diabetes care. Based on the registry in 2018, the total number of male patients with T2DM was 2,352.

Participants for this study were men with T2DM, who came for their clinical visit during the data collection period (one day in a week) and claimed not to have ED. Those who answered "No" to the question: "Do you think you have erection problem?" were invited to participate in this study. Those with self-perception of having ED were not included in this study, because it was not logical to evaluate their risk as they have already perceived having ED. The exclusion criteria were patients who required urgent treatment during their clinic visit and those who could not read English or Bahasa Malaysia.

This study used a bilingual self-administered questionnaire, which consisted of four sections (A-D) and a clinical data collection sheet. The participants took about 10-15 minutes to complete the questionnaire. Section A assessed participant sociodemographic data such as age, ethnicity, education level, employment status, and total monthly household income. Section B assessed risk perception for developing ED, which comprises two domains: (1) perceived susceptibility of ED (1 item) and (2) perceived severity of ED ( 3 items). Section C assessed participant knowledge of the risk factors of ED (12 items). Section D assessed their erectile function using the International Index of Erectile Function-5 items questionnaire (IIEF-5()). The clinical data collection sheet captured data on participant weight and height (to calculate body mass index (BMI)), HbA1c and total cholesterol within one year, smoking status, and comorbidities by reviewing their electronic medical records.

The perceived susceptibility of ED was assessed with the question, "What do you think your chances of getting erectile dysfunction within five years from now?". The response was from the options of the five-point Likert scale and the corresponding scores were "no chance" $=1$, "slight chance" $=2$, "moderate chance" $=3$, "high chance" $=4$, and "very high chance" $=5$. The higher the score, the higher the perceived susceptibility. Meanwhile, the perceived severity of ED was measured through agreement to three statements: (1) "I think that ED is a severe problem", (2) "Compared to other illness, erectile dysfunction is a minor problem", (3) "Erectile dysfunction gives serious impact on me". These items had fivepoint Likert scale responses, ranging from "strongly disagree (score of 1) to "strongly agree" (score of 5). Question two was reversely scored. The total score for the perceived severity of ED ranged between 3 and 15 . The higher the score, the higher the perceived severity of ED.

Participant knowledge of ED risk factors was measured through 12 items with "yes", "no", or "do not know" answers. A score of 1 was given for a correct answer and 0 for an incorrect or "do not know" answer. The total score ranged between 0 and 12 . The higher the score, the higher the knowledge.

All the items for sections $\mathrm{B}$ and $\mathrm{C}$ were developed based on literature review, ${ }^{12-15} \mathrm{HBM},{ }^{8}$ and discussions with a family physician. The questionnaire's content validation was tested by a panel of experts consisting of two family physicians with a particular interest in men's health and one clinical psychologist. Subsequently, face validation was conducted on ten male patients of different ethnicities, educational backgrounds, and comorbidities. Construct validity and internal consistency were tested among 49 men with T2DM from June to August 2019 at a university-based primary care clinic. Exploratory factor analysis was performed for all four risk perception items for developing ED (section B) using Principal Axis Factoring with Direct Oblimin rotation. The analysis revealed only one construct, in which Item 1 , Item 2 , and 
Item 3 assessed the perceived severity of ED had a loading factor of $0.58,0.52$, and 0.73 , respectively. However, the item that assessed the perceived susceptibility of ED had a loading factor of 0.26 . Due to this, two domains of risk perception were measured independently: (1) the perceived susceptibility with one item and (2) the perceived severity with three items. The Cronbach's $\alpha$ for the section assessing perceived severity of ED and the knowledge section was 0.64 and 0.67 .

The IIEF-5@ questionnaire is a copyrighted tool by Pfizer Inc. to assess erectile function within the past four weeks. It comprises five items with 5- and 6-point Likert scale responses. A score of 0-5 was awarded to each item with the total score ranging between 1 and 25 . The total score can be categorized into (1) No ED (score 22-25), (2) Mild ED (score 17-21), (3) Mild to Moderate ED (score 12-16), (4) Moderate ED (score 8-11), and (5) Severe ED (score 1-7). In this study, ED's presence was defined as men with mild to severe ED (IIEF-5() scores of 1-21). Permission to use the IIEF-5@ was obtained from Pfizer Inc. Previous studies have demonstrated the accuracy of the IIEF-5@ in diagnosing ED compared to the self-reported claims of the sufferers. ${ }^{16,17}$

The sample size was calculated using a one-mean formula, as the dependent variables (perceived susceptibility and perceived severity) were continuous data. Authors estimated the sample size based on assumed mean sum score and standard deviation of perceived susceptibility of 2.61 and 1.2, respectively, and for perceived susceptibility of 9.71 and 2.8 , respectively. The calculated sample size was 154 . Authors recruited 184 participants to account for $20 \%$ of incomplete data.

Data analysis was performed using statistical software. Since all continuous variables were not normally distributed, the data were presented in the median and inter-quartile range (IQR). Categorical variables were described in frequency $(\mathrm{n})$ and percentage $(\%)$. The dependent variables were the perceived susceptibility and the perceived severity of ED. The associations between these dependent variables and the independent variables were examined using simple linear regression. The independent variables were age, ethnicity, educational level, employment status, monthly household income, smoking status, presence of hypertension, presence of dyslipidemia, total cholesterol, BMI, HbA1c, presence of ED based on IIEF-5@, and knowledge score. The independent variables with clinical significance or a p-value of $<$ 0.25 from the bivariate analysis were selected into multiple linear regression analysis to determine their independent association with the dependent variables. Dummy tables were created for categorical independent variables. The significance level was set at $\mathrm{p}$-value $<0.05$.

Approval to conduct the study was obtained from the Medical Research and Ethics Committee (MREC) of the
Ministry of Health Malaysia (NMRR-18-3896-45035) and the Research Ethics Committee of Universiti Kebangsaan Malaysia (UKM FF-2019-146). Permission was obtained from the Selangor Director of Health and Family Medicine Specialist in charge of the clinic. The participants were required to sign an informed consent before participating in the study. Their anonymity was maintained throughout the research process. All patients with ED, either through self-reporting during the eligibility screening or identification using the IIEF-5@ questionnaire, were briefly counseled about the availability of treatment and were referred to the treating team if the participants agreed.

\section{Results}

A total of 195 male patients with T2DM were screened for study eligibility. Ultimately, 184 consented patients who did not perceive to have ED were recruited for this study. However, four participants were excluded from the analysis, because they did not have HbA1c taken within the past year, which left only 180 participants for the analysis. The response rate was $97.8 \%$.

Table 1 shows the sociodemographic and clinical characteristics of the participants. The median (IQR) age of the participants was 58.0 (14.0) years. The proportions of Malay $(41 \%)$ and Chinese $(40 \%)$ were similar. The remaining participants were Indian (18.9\%). Less than two-thirds attained secondary education $(61 \%)$ or were employed $(58 \%)$. The median (IQR) total monthly household income was RM 2,000 (2675.0), which was considered a low-class income. Besides diabetes, most of them also had hypertension $(91 \%)$ or hypercholesterolemia (90\%). The median (IQR) HbA1c and total cholesterol levels were $7.2 \%(2.3 \%)$ and $4.7(1.7)$ $\mathrm{mmol} / \mathrm{L}$. About $87.3 \%$ were overweight or obese and only $27 \%$ were smokers. Even though all the participants claimed not having ED, 43.9\% (79/180) had ED when assessed using IIEF-5@ (Table 1). Among those who had ED, $40.5 \%(32 / 79)$ had moderate to severe ED.

About half of the participants (51.7\%) perceived having no chance of getting ED within five years, whereas $19.4 \%$ perceived their chance was slight (Figure 1). The median (IQR) score for perceived susceptibility was 1.0 (2.0), which indicates a slight chance of getting ED. The median (IQR) score for the perceived severity of ED was 10.0 (6.0), which indicates a higher than "neutral" perception of ED severity. There was no relationship between perceived susceptibility and perceived severity (Spearman's rho correlation: -0.008 , p-value $=0.292$ )

The median (IQR) of the total score for knowledge on ED risk factors was 9.0 (4.0). Most of the participants could correctly identify the risk factors of ED (Figure 2). However, the five least recognized risk factors were high cholesterol levels $(43.9 \%)$, heart problems $(41.7 \%)$, al- 
Table 1. Sociodemographic and Clinical Characteristics of the Participants $(N=180)$

\begin{tabular}{|c|c|c|c|}
\hline Variable & Category & n $(\%)$ & Median (IQR) \\
\hline Age (years)* & & & $58.0(14.0)$ \\
\hline \multirow{3}{*}{ Ethnicity } & Malay & $74(41.1)$ & \\
\hline & Chinese & $72(40.0)$ & \\
\hline & Indian & $34(18.9)$ & \\
\hline \multirow[t]{3}{*}{ Education level } & No/primary & $32(17.8)$ & \\
\hline & Secondary & $110(61.1)$ & \\
\hline & Tertiary & $38(21.1)$ & \\
\hline \multirow{2}{*}{ Employment status } & Unemployed & $76(42.2)$ & \\
\hline & Employed & $104(57.8)$ & \\
\hline Monthly household income (RM)* & & & $2,000.0(2,675.0)$ \\
\hline \multirow[t]{2}{*}{ Smoking status } & Non-smoker/former & $131(72.8)$ & \\
\hline & Active smoker & $49(27.2)$ & \\
\hline \multirow[t]{4}{*}{ Comorbidities } & Hypertension & $165(91.7)$ & \\
\hline & Hypercholesterolemia & $163(90.6)$ & \\
\hline & Ischemic heart disease & $2(1.1)$ & \\
\hline & Stroke & $1(0.6)$ & \\
\hline $\mathrm{HbA} 1 \mathrm{c}(\%)^{*}$ & & & $7.2(2.3)$ \\
\hline Total cholesterol $(\mathrm{mmol} / \mathrm{L}) *$ & & & $4.7(1.7)$ \\
\hline BMI $\left(\mathrm{kg} / \mathrm{m}^{2}\right)^{*}$ & & & $27.5(6.8)$ \\
\hline \multirow[t]{3}{*}{ BMI classification } & Underweight/normal $(\mathrm{BMI}<22.9$ ) & $23(12.8)$ & \\
\hline & Overweight (BMI 23-27.4) & & $66(36.7)$ \\
\hline & Obese $($ BMI $>27.5)$ & $91(50.6)$ & \\
\hline IIEF-5 score* & & & $23.0(9.0)$ \\
\hline \multirow[t]{2}{*}{ Presence of ED (based on IIEF-5) } & No ED & $101(56.1)$ & \\
\hline & Have ED & $79(43.9)$ & \\
\hline
\end{tabular}

Notes: $\mathrm{IQR}=$ Inter-quartilie Range; RM = Ringgit Malaysia; BMI = Body Mass Index; ED = Erectile

Dysfunction; *All continuous data were not normally distributed.

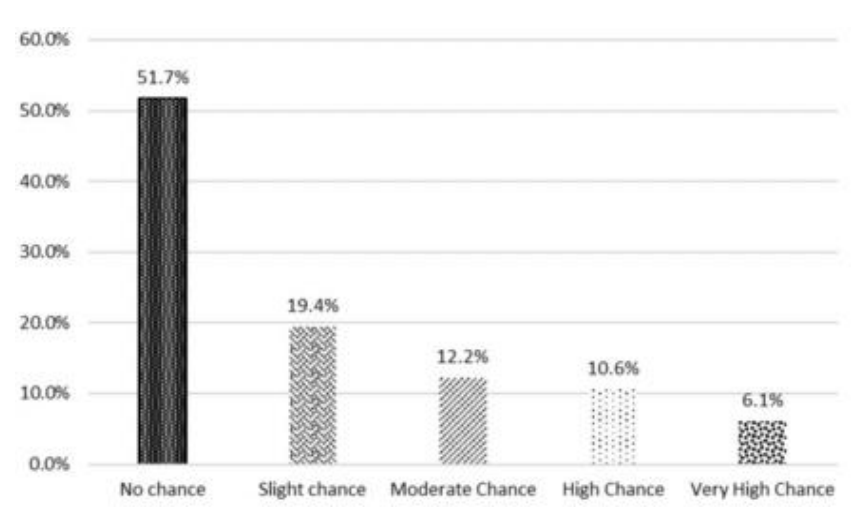

Figure 1. Participants' Perceived Susceptibility to Have Erectile Dysfunction $(\mathrm{N}=180)$

cohol $(41.7 \%)$, smoking cigarettes $(46.1 \%)$, and antihypertension medications $(41.1 \%)$.

Independent variables with clinical significance or a $p$-value of $<0.25$ identified using SLR were included in MLR to determine factors that significantly influenced perceived susceptibility or perceived severity of ED. For perceived susceptibility of ED, the variables were age, educational level, and presence of ED, whereas, for the perceived severity of ED, the variables were age, ethnicity, educational level, employment status, BMI, presence of actual ED, and knowledge of ED risk factors (Table 2). Only educational level (secondary education vs. no or primary education: $\mathrm{p}$-value $=0.045$; tertiary education

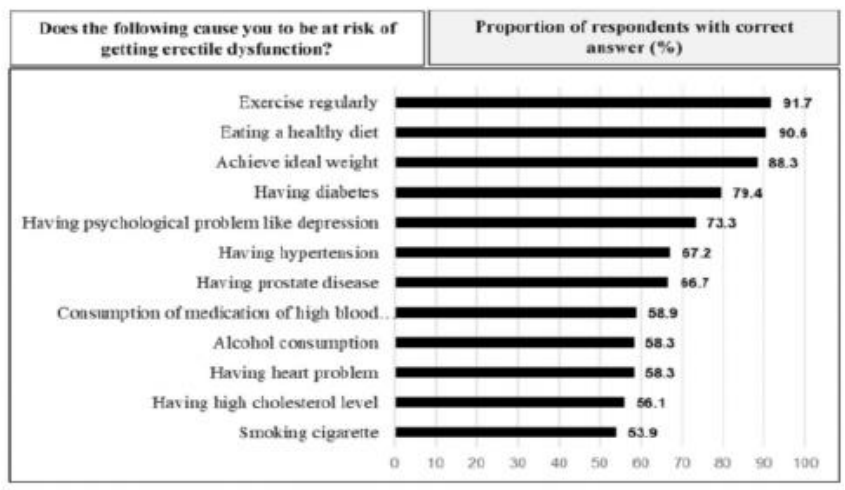

Total knowledge score: 12, Median: 9, Midpoint: 6

Figure 2. Participant Knowledge of Risk Factor of Erectile Dysfunction

vs. no or primary education: $\mathrm{p}$-value $=0.022$ ) and presence of ED ( $p$-value $<0.001$ ) were found to be significantly associated with perceived susceptibility of ED (Table 3). Those who attained secondary education or tertiary education had a significantly higher perceived susceptibility score than those who had no education or primary education by 0.19 and 0.21 , respectively. Similarly, participants with ED had significantly higher perceived susceptibility score by 0.30 compared to those who had no ED. For perceived severity of ED, the significant independent factors were ethnicity (Chinese vs. Malay: p-value $<0.001$ and Indian vs. Malay: p-value $<$ $0.001)$, employed $(p$-value $=0.026)$, and knowledge 
Table 2. Factors Associated with Perceived Susceptibility and Perceived Severity of Erectile Dysfunction (N = 180)

\begin{tabular}{|c|c|c|c|c|c|c|}
\hline \multirow{2}{*}{ Variable } & \multicolumn{3}{|c|}{ Perceived Susceptibility } & \multicolumn{3}{|c|}{ Perceived Severity } \\
\hline & Standardized $\beta$ & $95 \% \mathrm{CI}$ & p-value ${ }^{*}$ & Standardized $\beta$ & $95 \% \mathrm{CI}$ & p-value* \\
\hline Age (years) & 0.14 & $-0.01,0.04$ & 0.068 & -0.28 & $-0.14,-0.05$ & $<0.001$ \\
\hline Malay & 1 & & & 1 & & \\
\hline Chinese & -0.04 & $-0.51,0.32$ & 0.651 & -0.41 & $-3.58,-1.72$ & $<0.001$ \\
\hline Indian & -0.01 & $-0.53,0.51$ & 0.967 & -0.42 & $-4.54,-2.20$ & $<0.001$ \\
\hline $\mathrm{No} /$ primary education & 1 & & & 1 & & \\
\hline Secondary education & 0.21 & $0.05,1.05$ & 0.031 & 0.23 & $0.24,2.73$ & 0.020 \\
\hline Tertiary education & 0.20 & $0.03,1.22$ & 0.040 & 0.21 & $0.14,3.12$ & 0.032 \\
\hline Unemployed & 1 & & & 1 & & \\
\hline Employed & -0.07 & $-0.56,0.20$ & 0.344 & 0.23 & $0.54,2.39$ & 0.002 \\
\hline Monthly household income (RM) & 0.05 & $0.00,0.00$ & 0.543 & 0.14 & $0.00,0.00$ & 0.063 \\
\hline Non/former smoker & 1 & & & 1 & & \\
\hline Active smoker & -0.05 & $-0.56,0.28$ & 0.512 & 0.02 & $-0.92,1.20$ & 0.794 \\
\hline No hypertension & 1 & & & 1 & & \\
\hline Hypertension & 0.05 & $-0.46,0.90$ & 0.527 & -0.03 & $-2.06,1.34$ & 0.678 \\
\hline No hypercholesterolemia & 1 & & & 1 & & \\
\hline Hypercholesterolemia & 0.06 & $-0.38,0.90$ & 0.425 & 0.02 & $-1.35,1.86$ & 0.752 \\
\hline Total cholesterol $(\mathrm{mmol} / \mathrm{L})$ & 0.08 & $-0.07,0.23$ & 0.279 & -0.05 & $-0.51,0.24$ & 0.484 \\
\hline Body mass index $\left(\mathrm{kg} / \mathrm{m}^{2}\right)$ & -0.01 & $-0.04,0.03$ & 0.875 & 0.15 & $0.01,0.16$ & 0.047 \\
\hline $\operatorname{HbA1c}(\%)$ & 0.02 & $-0.09,0.12$ & 0.772 & 0.13 & $-0.03,0.47$ & 0.089 \\
\hline No erectile dysfunction (based on IIEF-5) & 1 & & & 1 & & \\
\hline Presence of erectile dysfunction & 0.32 & $0.45,1.17$ & $<0.001$ & -0.18 & $-2.11,-0.25$ & 0.013 \\
\hline Knowledge score & 0.07 & $-0.04,0.10$ & 0.346 & 0.39 & $0.30,0.61$ & $<0.001$ \\
\hline
\end{tabular}

Notes: *Significance: $\mathrm{p}$-value $<0.05 ; \mathrm{CI}=$ Confidence Interval; RM = Ringgit Malaysia

Table 3. Multiple Linear Regression: Factors Associated with Perceived Susceptibility and Perceived Severity of Erectile Dysfunction

\begin{tabular}{|c|c|c|c|c|c|c|}
\hline \multirow{2}{*}{ Variable } & \multicolumn{3}{|c|}{ Perceived Susceptibility ${ }^{a}$} & \multicolumn{3}{|c|}{ Perceived Severity ${ }^{\mathbf{b}}$} \\
\hline & Standardized $\beta$ & $95 \% \mathrm{CI}$ & p-value* & Standardized $\beta$ & $\mathbf{9 5} \% \mathbf{C I}$ & p-value \\
\hline Age (years) & 0.09 & $-0.01,0.03$ & 0.236 & -0.11 & $-0.09,0.01$ & 0.151 \\
\hline Malay & & & & 1 & & \\
\hline Chinese & & & & -0.32 & $-2.98,-1.14$ & $<0.001$ \\
\hline Indian & & & & -0.31 & $-3.70,-1.36$ & $<0.001$ \\
\hline No/primary education & 1 & & & 1 & & \\
\hline Secondary education & 0.19 & $0.01,0.97$ & 0.045 & 0.08 & -0.561 .60 & 0.345 \\
\hline Tertiary education & 0.21 & $0.10,1.23$ & 0.022 & 0.05 & $-1.01,1.83$ & 0.568 \\
\hline Unemployed & & & & 1 & & \\
\hline Employed & & & & 0.17 & $0.14,2.10$ & 0.026 \\
\hline Monthly household income (RM) & & & & -0.03 & $0.00,0.00$ & 0.663 \\
\hline Body mass index $\left(\mathrm{kg} / \mathrm{m}^{2}\right)$ & & & & 0.04 & $-0.05,0.10$ & 0.516 \\
\hline $\mathrm{HbA} 1 \mathrm{c}(\%)$ & & & & 0.10 & $-0.05,0.38$ & 0.128 \\
\hline No erectile dysfunction (based on IIEF-5) & 1 & & & 1 & & \\
\hline Presence of actual erectile dysfunction & 0.30 & $0.39,1.13$ & $<0.001$ & -0.10 & $-1.49,0.16$ & 0.114 \\
\hline Knowledge score & & & & 0.27 & $0.16,0.47$ & $<0.001$ \\
\hline
\end{tabular}

Notes: "Significance: $\mathrm{p}$-value $<0.05 ; \mathrm{CI}=$ Confidence Interval; $\mathrm{RM}=$ Ringgit Malaysia

a Variables included in multiple linear regression (MLR) for perceived susceptibility: Age, Education, Presence of actual erectile dysfunction (ED); R² for perceived susceptibility: 0.135; MLR using enter method

bVariables included in MLR for perceived severity: Age, Ethnicity, Education, Employment status, Monthly household income, BMI, HbA1c, Presence of actual ED, Knowledge score; R2 for perceived severity: 0.377; MLR using enter method.

score (p-value < 0.001) (Table 3). Chinese and Indians had lower perceived severity scores compared to Malay by 0.32 and 0.31 , respectively. Employed men had a higher perceived severity score by 0.17 compared with unemployed. For every increment of 1 score in knowledge, there was an increase in perceived severity score by 0.27 .

\section{Discussion}

In this study, assessing perceived susceptibility and perceived severity of ED among men with T2DM could explain how these patients appreciated their risk to develop ED. Participants in this study were mainly middleaged Malay and Chinese men with secondary education and low socioeconomic classes. Apart from having T2DM as a risk factor of ED, many had other risk factors, 
including hypertension, dyslipidemia, and overweight or obesity.

It is found that $43.9 \%$ of the participants did not seem aware of their ED condition. In fact, many reported that they did not have ED but had moderate to severe ED. A similar discrepancy between self-reported ED and the presence of ED assessed using IIEF-5 was observed in a local study. ${ }^{18}$ These findings suggest under-reporting of ED by the participants, which can be due to socially-biased responses when asked by the investigator about erection problems during eligibility screening. They could also underestimate the ED symptoms they experienced and did not consider themselves as having ED to preserve their masculine image. 19,20 Misunderstandings about ED as a total loss of response and inability to perform a sexual activity could make them feel that they did not have ED, especially if they were still able to initiate sex.4,21 There was a possibility that these men might have confused ED with other types of male sexual dysfunction, namely premature or retrograde ejaculation.

According to the HBM, people should feel susceptible to certain diseases by evaluating their susceptibility to contracting the disease and understanding the negative consequences. If they perceive susceptibility to and the severity of the disease, they could appreciate the risk and, thus, feel threatened and vulnerable. Generally, many participants had an inaccurate perception of susceptibility, as $71.1 \%$ had no or slight perception of susceptibility. The median score for perceived susceptibility was only 1.0, equivalent to a slight chance of getting ED. However, their perception of ED severity was moderate. It appears that they were aware of the severity and the impacts of ED, but felt less vulnerable to suffering from $\mathrm{ED}$, which was contrary to our hypothesis. Insufficient appreciation of vulnerability could be due to their optimistic bias or unrealistic optimism, which is a positive belief of being less likely to experience health risks. ${ }^{22}$ Men tend to underestimate their risk of having ED and ignore its importance to maintain their masculine and macho identities as having ED is often portrayed as being weak. ${ }^{19,20}$ This unrealistic optimism is quite worrying because it might lead to poor health-seeking behavior. ${ }^{23}$ The unique relationship between perceived susceptibility and perceived severity of ED in this study, which was shown to be non-significant, may also indicate the representation of different concepts with what is conceptualized by the HBM. This complex relationship was also demonstrated by El-Toukhy, et al. ${ }^{24}$

The moderate level of perceived severity of ED in the participants was consistent with other local studies, ${ }^{12,19}$ which highlight that men with T2DM realized the seriousness of ED. They appreciated its negative impact on QOL and on the relationship with their partner. ${ }^{12,19}$ Nevertheless, no previous study assessed the perception of susceptibility to develop ED among patients with T2DM; therefore, it is difficult to compare and interpret the significance of our findings. There was a nearly similar study, but it was conducted among different populations, e.g., patients with localized prostate cancer. ${ }^{25}$ The study assessed the risk perception to develop ED due to various prostate cancer treatments. An inaccurate perception of susceptibility was found to be expected, which was thought to be due to the lack of information provided during counseling. ${ }^{25}$

Since most of the participants could correctly recognize most of the risk factors of ED and their average total score was above the midpoint of the range score, their knowledge could generally be regarded as acceptable. The knowledge of ED risk factors was significantly associated with participant perception of ED severity, emphasizing the importance of knowledge in influencing one's risk perception. Our study's acceptable level of knowledge was similar to local research, but different from overseas studies conducted in Switzerland and Poland. ${ }^{12,26,27}$ In the Swiss study, about half of their participants could not name even one single risk factor of ED, 26 whereas the study in Poland showed that twofifths of high-risk male patients were unable to name any modifiable risk factors for ED, including smoking, diabetes, hyperlipidemia, hypertension, obesity, and physical inactivity. ${ }^{27}$ Only $6 \%$ could list all six of the risk factors correctly. ${ }^{27}$ Good knowledge among these high-risk patients could be due to health education provided by healthcare providers. ${ }^{12}$ In the era of the internet, the patients might have been exposed to ED information from various social media and websites. ${ }^{26}$ Level of education may also influence their knowledge and appreciation of risk. In this study, those with higher education were significantly associated with a higher level of perceived susceptibility, which further emphasized the role of intellect in appreciating risk. Nevertheless, our study also demonstrated the gaps in knowledge among participants that need to be addressed during counseling on ED risk factors: smoking, alcohol, anti-hypertensive medications, heart disease, and high cholesterol.

Compared to those who did not have ED assessed using IIEF-5(), the participants who actually had ED perceived higher susceptibility to getting ED in the next five years. With the presence of symptoms, they could appreciate their vulnerability, but the experience might not be enough to make them aware of their ED. These findings suggest that they may have under-recognized or underestimated the symptoms that they experienced. Men might normalize the condition as part of the aging process. ${ }^{19}$ This normalization was observed in a study conducted in the United Kingdom, US, France, Germany, Italy, and Spain, which revealed that men with ED did not seek treatment as they believed ED is a normal part of the ag- 
ing process. ${ }^{28}$ Another explanation could be due to hegemonic masculinity identity demonstrated by men, in which they denied ED symptoms as it implies weakness. ${ }^{22}$

As suggested by our findings, the perception of ED severity may also be influenced by culture, which is in line with Low, et al., ${ }^{4}$ in demonstrating differences in ethnicity perception. In the study, Malays regarded ED as an illness and felt that the impacts of ED on their relationship with their spouse were significant, and were thus motivated to seek treatment. However, Chinese and Indian perceptions of ED imply that ED was a less severe problem. The Chinese believed that psychological problems, low self-esteem, and anxiety were the cause of ED, and they tended to be more accepting if it was due to aging. Indians attributed ED to fate and experienced less impact on their relationship.

To date, this study was among the initial studies conducted in Malaysia that examined risk perception of ED among men with T2DM using both domains of risk perception, e.g., perceived susceptibility and perceived severity. Therefore, this study's findings could reveal the complex connection between perceived severity and perceived susceptibility to informing one's risk perception. The findings also highlight the importance of knowledge on the risk factor of ED, thus education should be provided on risk factors. This study identifies the underrecognition or underestimation of ED symptoms. Therefore, men with T2DM should be educated on the symptoms of ED to ensure good health-seeking behavior. The findings could also provide essential information for future studies.

The present study had several limitations. First, the recruitment of men who did not perceive having ED was made through a single direct question: "Do you think you have erection problem?". This question could result in social desirability biased responses, which may have led to imprecision in recruiting of the sample population. This effect could be minimized by using a self-administered screening tool. Secondly, the convenience sampling of this study limits the generalization of the findings due to selection bias. Probabilistic sampling should be considered for upcoming research. Thirdly, this study used one item to assess ED's perceived susceptibility, reducing the item's ability to capture and fully represent the construct. Future studies should develop a tool with multiple items that can validly assess perceived susceptibility. Fourthly, a self-administered questionnaire could lead to response bias and recall bias, which might influence findings' accuracy.

\section{Conclusion}

Generally, many men with T2DM who claimed to have no ED had an inaccurate perception of susceptibility to develop ED in five years. Besides, their perception of ED severity was only moderate. These findings imply poor risk perception among them, which was significantly influenced by cultural and socioeconomic factors, education attainment, knowledge on ED risk factors, and presence of ED symptoms. Under-recognition or underestimation of ED symptoms was observed as many did experience moderate to severe ED symptoms.

\section{Recommendation}

Thus, risk perception of ED among men with T2DM should be assessed in a clinical setting to identify their misperception. Appropriate counseling and education should also be provided to improve their risk perception. Future studies should consider both domains of risk perception of ED (perceived susceptibility and perceived severity) in other populations to improve our understandings of the complex interactions between the two domains informing one's risk perception.

\begin{abstract}
Abbreviations
ED: Erectile dysfunction; T2DM: Type 2 diabetes mellitus; HBM: Health Belief Model; QOL: Quality of Life; IIEF-5@: International Index of Erectile Function-5 items questionnaire; BMI: Body mass index; MLR: Multiple logistic regression; IQR: Inter-quartile range.
\end{abstract}

Ethics Approval and Consent to Participate

Approval to conduct the study was obtained from the Medical Research and Ethics Committee (MREC) of the Ministry of Health Malaysia (NMRR-18-3896-45035) and the Research Ethics Committee of Universiti Kebangsaan Malaysia (UKM FF-2019-146).

\section{Competing Interest}

The author declares that there is no significant competing financial, professional, or personal interest that might have affected the performance or presentation of the work described in this manuscript.

\section{Availability of Data and Materials}

Data and materials of this study can be obtained upon request.

\section{Authors' Contribution}

AM: designed, developed the study tool, collected and analyzed data, prepared the manuscript; HT: designed, developed the study tool, analyzed data, prepared the manuscript, revised the manuscript; SA: developed the study tool, reviewed and revised the manuscript; SFT: reviewed and revised the manuscript; MA: reviewed and revised the manuscript. All authors reviewed and approved the final manuscript.

\section{Acknowledgment}

We would like to thank the Director-General of Health Malaysia for his permission to publish this paper. Special thanks to the Family Medicine Specialist of the health clinic and all clinic staff who had given their support and assistance. 


\section{References}

1. Burnett AL, Nehra A, Breau RH, Culkin DJ, Faraday MM, Hakim LS, et al. Erectile dysfunction: AUA guideline. The Journal of Urology. 2018; 200 (3): 633-41.

2. Colson MH, Cuzin B, Faix A, Grellet L, Huyghes E. Current epidemiology of erectile dysfunction, an update. Sexologies. 2018; 27 (1): e713.

3. Huri HZ, Mat Sanusi ND, Razack AHA, Mark R. Association of psychological factors, patients' knowledge, and management among patients with erectile dysfunction. Patient Prefer Adherence 2016; 10: 807-23.

4. Low WY, Wong YL, Zulkifli SN, Tan HM. Malaysian cultural differences in knowledge, attitudes and practices related to erectile dysfunction: focus group discussions. International Journal of Impotence Research. 2002; 14: 440-5.

5. Ab Rahman AA, Al-Sadat N, Yun Low W. Prevalence of erectile dysfunction in primary care setting, Malaysia. Journal of Men's Health. 2011; 8 (Suppl 1): S50-3.

6. Penson DF, Latini DM, Lubeck DP, Wallace KL, Henning JM, Lue TF. Do impotent men with diabetes have more severe erectile dysfunction and worse quality of life than the general population of impotent patients?: results from the exploratory comprehensive evaluation of erectile dysfunction (EXCEED) database. Diabetes Care. 2003; 26: 10939.

7. Stimac G. Erectile dysfunction. Medicus. 2012; 21: 87-93.

8. Luger TM. Health beliefs/health belief model. In: Gellman M.D., Turner J.R. Encyclopedia of behavioral medicine. New York, NY: Springer; 2013.

9. Shreck E, Gonzalez JS, Cohen HW, Walker EA. Risk perception and self-management in urban, diverse adults with type 2 diabetes: the improving diabetes outcomes study. International Journal of Behavioral Medicine. 2014; 21: 88-98.

10. Pereira MG, Pedras S, Ferreira G. Self-reported adherence to foot care in type 2 diabetes patients: do illness representations and distress matter?. Primary Health Care Research \& Development. 2018; 20: 1-8.

11. Rouyard T, Kent S, Baskerville R, Leal J, Gray A. Perceptions of risks for diabetes-related complications in type 2 diabetes populations: a systematic review. Diabetic Medicine. 2017; 34: 467-77.

12. Gillani SW, Sulaiman SAS, Sari YO. Population based survey on knowledge and quality of life perception on erectile dysfunction among diabetic and non-diabetic attributes, Penang, Malaysia. International Journal of Pharmacy and Pharmaceutical Sciences. 2011; 3: 229-34.

13. Kim C, McEwen LN, Piette JD, Goewey J, Ferrara A, Walker EA. Risk perception for diabetes among women with histories of gestational diabetes mellitus. Diabetes Care. 2007; 30: 2281-6.

14. Fuster-RuizdeApodaca MJ, Laguia A, Molero F, Toledo J, Arrillaga A, Jaen A. Psychosocial determinants of HIV testing across stages of change in Spanish population: a cross-sectional national survey. BMC Public Health. 2017; 17: 234.
15. Kurz M. Sexual dysfunctions: erectile dysfunction and premature ejaculation. Journal fur Urologie und Urogynakologie 2013; 20: 9-10.

16. Reis MM, Abdo CH. Prevalence of erectile dysfunction as defined by the International Index of Erectile Function (IIEF) and self-reported erectile dysfunction in a sample of Brazilian men who consider themselves healthy. Journal of Sex \& Marital Therapy. 2010; 36: 87-100.

17. Song WH, Park J, Yoo S, Oh S, Cho SY, Cho MC, et al. Changes in the prevalence and risk factors of erectile dysfunction during a decade: the Korean internet sexuality survey (KISS), a 10-year-interval webbased survey. World Journal of Men's Health. 2019; 37: 199-209.

18. Lo WH, Fu SN, Wong CKH, Chen ES. Prevalence, correlates, attitude and treatment seeking of erectile dysfunction among type 2 diabetic Chinese men attending primary care outpatient clinics. Asian Journal of Andrology. 2014; 16: 755-60.

19. Courtenay WH. Constructions of masculinity and their influence on men's well-being: a theory of gender and health. Social Science \& Medicine. 2000; 50: 1385-401.

20. Connell RW, Messerschmidt JW. Hegemonic masculinity: rethinking the concept. Gender \& Society. 2005; 19: 829-59.

21. Low WY, Ng CJ, Choo WY, Tan HM. How do men perceive erectile dysfunction and its treatment? a qualitative study on opinions of men. Aging Male. 2006; 9: 175-80.

22. Radcliffe N, Klein W. Dispositional, unrealistic, and comparative optimism: differential relations with the knowledge and processing of risk information and beliefs about personal risk. Personality and Social Psychology Bulletin. 2002; 28: 836-46.

23. Ahmad Arifin S, Mat Yusuf A, Ili Nabilah R, Md Isa L. Health seeking behavior of health disorders of men among government servants in Kuantan: prevalence study towards a healthy ummah. International Medical Journal Malaysia. 2018; 17: 157-64.

24. El-Toukhy S. Parsing susceptibility and severity dimensions of health risk perceptions. Journal of Health Communication. 2015; 20: 499511.

25. van Stam MA, van der Poel HG, van der Voort van Zyp JRN, Tillier $\mathrm{CN}$, Horenblas S, Aaronson NK, et al. The accuracy of patients' perceptions of the risks associated with localized prostate cancer treatments. BJU International. 2018; 121: 405-14.

26. Baumgartner MK, Hermanns T, Cohen A, Schmid DM, Seifert B, Sulser T, et al. Patients' knowledge about risk factors for erectile dysfunction is poor. The Journal of Sexual Medicine. 2008; 5: 2399-404.

27. Kałka D, Domagała Z, Rakowska A, Womperski K, Franke R, Sylwina-Krauz E, et al. Modifiable risk factors for erectile dysfunction: an assessment of the awareness of such factors in patients suffering from ischaemic heart disease. International Journal of Impotence Research. 2016; 28: 14-9.

28. Shabsigh R, Perelman MA, Laumann EO, Lockhart DC. Drivers and barriers to seeking treatment for erectile dysfunction: a comparison of six countries. BJU International. 2004; 94: 1055-65. 